\title{
Germanica
}

\section{La transfiguration suspecte d'un bouc émissaire : Walter Rathenau vu par Ernst von Salomon}

W. Rathenau als Sündenbock in E. von Salomons Werk

The dubious métamorphose of a scapegoat: Walter Rathenau seen by Ernst von

Salomon in The Outlaws and The Answers

\section{Pierre Giraud}

\section{(2) OpenEdition}

\section{Journals}

\section{Édition électronique}

URL : http://journals.openedition.org/germanica/2778

DOI : 10.4000/germanica.2778

ISSN : 2107-0784

\section{Éditeur}

Université de Lille

\section{Édition imprimée}

Date de publication : 31 décembre 1987

Pagination : 81-98

ISSN : 0984-2632

\section{Référence électronique}

Pierre Giraud, «La transfiguration suspecte d'un bouc émissaire : Walter Rathenau vu par Ernst von

Salomon », Germanica [En ligne], 2 | 1987, mis en ligne le 16 février 2015, consulté le 06 octobre 2020. URL : http://journals.openedition.org/germanica/2778; DOI : https://doi.org/10.4000/germanica.2778

Ce document a été généré automatiquement le 6 octobre 2020.

(c) Tous droits réservés 


\title{
La transfiguration suspecte d'un bouc émissaire : Walter Rathenau vu par Ernst von Salomon
}

\author{
W. Rathenau als Sündenbock in E. von Salomons Werk \\ The dubious métamorphose of a scapegoat: Walter Rathenau seen by Ernst von \\ Salomon in The Outlaws and The Answers
}

Pierre Giraud

$\mathrm{Si}$, dans les premières années de la République de Weimar, un homme politique cristallise autour de sa personne pour des raisons essentiellement ethniques la haine des milieux conservateurs et ultranationalistes, c'est bien Walther Rathenau. Industriel, président de l'AEG, essayiste reconnu, membre du Parti démocrate allemand, il devient ministre de la Reconstruction dans le cabinet Wirth de mai à octobre 1921, puis ministre des Affaires étrangères en janvier 1922, toujours dans le cabinet Wirth. Accusé plus ou moins violemment par les milieux qui lui sont hostiles de trahir les intérêts supérieurs de la nation allemande, il est assassiné le 24 juin 1922 par de jeunes nationalistes d'extrême-droite. Un écrivain, Ernst von Salomon, dont la notoriété ${ }^{1}$ a toujours été plus grande en France qu'en Allemagne, s'est trouvé mêlé à l'assassinat de Rathenau et fut condamné pour complicité à cinq ans de réclusion. Dans deux de ses œuvres qui se veulent ouvertement autobiographiques et documentaires, Les Réprouvés (Die Geächteten, publié en 1930) et Le Questionnaire (Der Fragebogen, publié en 1951)², Salomon revient sur les circonstances et les motivations de cet acte, soucieux de témoigner pour une "génération perdue " et de redresser certaines falsifications postérieures aux faits. Dans la lettre-préface où, en 1961, il donne son accord à la réédition non retouchée des Réprouvés dans la collection de poche Rowohlt, il écrit que l'assassinat de Rathenau constitue bien le point central du livre. Son objectif est d'en donner non pas une justification, mais une description dans la mesure où, selon lui, le procès n'avait pas permis d'en éclairer les mobiles. Or quelle n'est pas la surprise du lecteur de constater que la haine n'est pas au rendez-vous dans l'image qu'Ernst von Salomon brosse de Rathenau. Le bouc émissaire est transfiguré en héros. Gomme l'écrit 
Roger Stéphane dans un article récent ${ }^{3}$, «le meurtre de Rathenau est le seul assassinat politique suscité par l'admiration ». Jugement paradoxal qui nous parait souscrire un peu vite à la vision de l'auteur. Il mérite en tout cas examen et amène à s'interroger sur les composantes et les arrière-pensées d'un portrait qui semble relever davantage de la fabrication que du souci de la vérité ou du témoignage rigoureux.

On constate tout d'abord qu'Ernst von Salomon apporte un grand soin à suggérer à son lecteur que Rathenau n'a pas été assassiné pour des motifs vulgaires tels, par exemple, que l'antisémitisme. À l'en croire, l'antisémitisme n'a pas joué le moindre rôle dans le choix de la victime, ce qui est une manière oblique de réhabiliter partiellement les assassins et leurs complices. Salomon est trop habile pour affirmer ouvertement cette thèse. Jamais il n'est dit explicitement dans Les Réprouvés que l'assassinat de Rathenau ne fut pas inspiré par l'antisémitisme. Il est tout de même quelque peu curieux qu'à aucun moment ne soit mentionnée l'appartenance de Rathenau à la communauté juive. À une époque où quantité de nationalistes dénonçaient en Allemagne les méfaits de "l'Internationale juive », pareille omission de la part de quelqu'un pour qui la nation est précisément la référence absolue paraît insolite.

D'autres indices sont également révélateurs. Soucieux d'individualiser ses compagnons de route, Salomon souligne qu'ils ne sauraient être confondus avec les hommes de main d'autres groupes nationalistes. Essentiellement en raison de leurs mobiles qui sont élevés. On a affaire à des héros. Or l'antisémitisme ne sied pas aux héros. Pour Salomon il est tout juste bon pour la masse avide d'explications courtes et basses. Au mieux il peut faire office de diversion. Ainsi, au moment où il se sépare de ses complices peu avant l'exécution de l'attentat, Kern, auquel Salomon demande ce qu'il conviendra de déclarer à la police en cas d'arrestation, a une réponse toute prête. Qu'ils déclarent par exemple que la victime était un des Sages de Sion ou qu'il s'apprêtait à marier sa sœur au bolchevique Radek ${ }^{4}$. On sait en effet que certains journaux d'extrême-droite diffuseront ce genre de "révélations». Sans renier l'opportunité momentanée d'une telle stratégie, Salomon prend néanmoins ses distances. Ce n'est pas contre le juif Rathenau que les coups ont été portés.

4 Ne risque-t-on pas d'objecter qu'à l'époque l'antisémitisme était le principal cheval de bataille des associations nationalistes, le fondement même de leur propagande et que donc une telle présentation des faits paraît difficilement plausible? Salomon semble avoir prévu l'objection. Il y répond par une anecdote dont Hambourg fournit le cadre et qui se rattache au moment où, en compagnie de Kern, il nouait des contacts avec toutes sortes de groupes nationalistes afin de recruter des exécutants prêts à tout. C'est ainsi qu'un soir, au cours d'une réunion organisée par une association patriotique, il fit la connaissance d'un membre du bureau du "deutsch-völkischer Schutzund Trutzbund » qui lui remet toute une série de brochures antisémites et l'entretient des fameux secrets des Sages de Sion. Croyant avoir gagné un nouvel adepte à sa cause, ce jeune nationaliste s'apprête à lui remettre une carte d'adhésion, mais, à l'écoute du nom de Salomon, il se ravise et se détourne de lui sans lui remettre de carte. Apparemment il a pris Salomon pour un juif ${ }^{5}$. Ainsi suggère-t-on au lecteur qu'une victime - oh! très relative - de l'antisémitisme ne pouvait trouver là une source d'inspiration à son action.

5 Le souci chez Salomon de se laver de tout soupçon d'antisémitisme semble d'ailleurs tourner à l'obsession'. Dans Le Questionnaire, le lecteur apprend vers la fin du récit que la compagne avec laquelle il a vécu maritalement durant toute la période du nazisme, 
Ille, était juive. Lors des dernières élections libres de la République de Weimar, elle a donné sa voix au Parti national-socialiste. Durant toute la période hitlérienne, elle ne sera jamais inquiétée. Salomon non plus d'ailleurs. Or le régime ne badinait guère sur ces questions-là. Ordinairement prodigue de détails sur sa vie privée, l'auteur reste curieusement muet sur l'explication de ce phénomène. Fiction? Vérité ? Jeu trouble en tout cas.

6 La hantise de se voir taxer d'antisémitisme est si forte chez lui qu'elle le poussera même à confier qu'il a du sang juif dans les veines. C'est en tout cas la confidence qu'il fera au rédacteur en chef du journal libéral Berliner Tageblatt, Ernst Feder, lui-même juif. Ce dernier rapporte en effet dans ses Mémoires ${ }^{7}$ qu'il reçut la visite d'Ernst von Salomon en juillet 1929, donc au moment où ce dernier, sorti de prison, travaillait à la rédaction des Réprouvés. Après avoir confié que désormais il « écrivaillait » et qu'il était bien décidé à mener la vie d'un honnête homme, Salomon souligna qu'« à son avis, le meurtre n'avait pas frappé (en Rathenau) le juif, mais le représentant de la République » et qu'il ne "songeait pas à nier que lui-même, comme d'ailleurs l'indiquait son nom, avait du sang juif dans les veines $»^{8}$. Etrange façon de débuter une vie d'honnête homme, au moins sur le plan intellectuel. On le sait, Salomon ne manque pas d'imagination et ne lésine pas sur la délicatesse des moyens dès lors qu'il s'agit d'atteindre une fin précise : ici démontrer que Rathenau n'a pas été exécuté en raison de ses origines juives.

7 Au moment de corriger les épreuves des Réprouvés, Salomon constata que son livre ne contenait pas le moindre propos antisémite. «De fait », écrit-il, « Rathenau fut le seul juif que nous exécutâmes $»^{9}$. L'absence d'antisémitisme n'est donc pas le fruit d'une intention délibérée de l'auteur. C'est un fait objectif. "Pourtant nous étions tous antisémites", fait remarquer Plaas, l'un des comparses. Salomon le concède et reconnaît que le mouvement nationaliste dans la totalité de ses composantes était antisémite. Mais c'est pour ajouter tout aussitôt qu'avec Rathenau la question n'est pas simple. N'a-t-il pas pris lui-même sur la question juive des positions bien particulières qui avaient déchaîné chez ses semblables une véritable tempête? N’a-t-il pas parlé d'une horde asiatique établie sur le sol de la Marche de Brandebourg? N'a-t-il pas repris lui-même certains thèmes antisémites et déploré l'insuffisante assimilation des juifs allemands? Salomon fait allusion ici à l'article que Rathenau avait publié sous un pseudonyme dans la revue de Maximilian Harden, Die Zukunft ${ }^{10}$. Salomon se garde bien de préciser que Rathenau avait plus tard renié cet article et qu'il ne l'avait pas repris dans son premier livre Reflexionen publié en 1908. Rathenau n'a-t-il pas écrit par ailleurs - Salomon se réfère ici à certaines lettres ${ }^{11}$ que le président de l'AEG avait échangées avec son ami Wilhelm Schwaner - qu'à ses yeux les juifs constituaient une peuplade allemande comme les autres, que si, d'un côté, il se sentait plus éloigné d'eux que des habitants du Brandebourg ou du Schleswig-Holstein, de l'autre il s'en sentait plus proche que des Saxons ou des Bavarois? Bref, pour Salomon, il s'agit en se réclamant de la caution de Rathenau de montrer que ce dernier avait pris soin de ne pas s'identifier pleinement à la communauté juive. Dans le même passage du Questionnaire, Salomon rapporte une brève conversation avec Ernst Jünger dont le silence, lors de la parution des Réprouvés, l'avait étonné et déçu. C'est pourquoi, dit-il, il eut l'insolence de lui demander ce qu'il en pensait. Jünger lui reprocha alors de n'avoir pas eu le courage d'écrire que Rathenau avait été assassiné parce qu'il était juif ${ }^{12}$. À Jünger comme aux juifs de Palestine qui lui adressaient alors des lettres identiques, Salomon oppose la même réponse : parce que ce n'est pas vrai. Non pas qu'à ses yeux - la réflexion qui va 
suivre est proposée après 1945 - l'antisémitisme ne puisse constituer, le cas échéant, une conviction politique, même si elle n'est pas de bon ton. "Mais ce n'était pas vrai. Rathenau ne fut pas tué parce qu'il était juif $»^{13}$.

Salomon laisse alors à son ami Plaas le soin de compléter la thèse : Rathenau fut tué bien que juif. Pourquoi cette restriction? On pouvait craindre, dit-il, que l'opinion ne se méprît sur les véritables motivations des assassins, comme tel fut en effet le cas. Plaas tenta même de convaincre son chef, Kern, du bien-fondé de cette objection. Peine perdue. La thèse est poussée là à son paroxysme : la qualité de juif aurait protégé - un temps - Rathenau. Cette insistance reprise à plus de vingt ans de distance est symptomatique, même s'il ajoute dans le même passage du Questionnaire: «Mes sympathies et mes antipathies ne furent jamais de nature biologique $»^{14}$. De quels sentiments étaient-ils donc animés à l'égard de Rathenau, lui et ses compagnons?

Après l'attentat et lors du procès qui suivit, plusieurs journalistes avaient souligné que les jeunes gens impliqués dans l'affaire avaient été le jouet de leurs instincts les plus vils et semblaient tout à fait dépourvus de culture, en particulier de culture politique. Ils avaient ajouté - et la lettre adressée à la mère de l'un d'eux, Ernst Werner Techow, par la mère de Rathenau allait dans le même sens - que, s'ils avaient connu leur victime, ils auraient banni de leur esprit toute idée de crime. Si Salomon met beaucoup d'application à détacher Rathenau de sa condition de juif et à laver l'attentat de toute tache antisémite, il en met tout autant à suggérer que lui et ses compagnons connaissaient parfaitement l'homme dont ils avaient décidé la mort. L'image de Rathenau présentée dans Les Réprouvés est un hommage rendu au penseur, à l'écrivain, à l'homme politique et d'abord à l'homme tout court. Rarement assassins auront eu autant de considération et de respect pour leur victime. À en croire Salomon, ce respect et cette considération reposent sur une connaissance approfondie du personnage, de ses actes, de ses écrits et, pour commencer, sur une connaissance directe, physique, de l'individu.

En effet, les assassins ont eu plusieurs occasions d'approcher directement ou indirectement leur future victime et les impressions qu'ils ont retirées de ces rencontres ont toujours été très flatteuses. Dans le chapitre des Réprouvés intitulé « $\mathrm{O}$. G. », Salomon évoque longuement une intervention que Rathenau fit le 29 octobre 1921 à la maison de la culture populaire de Francfort pour le compte du Parti démocrate. Salomon s'y est rendu en compagnie de Kern. Gomme à leur arrivée la salle était déjà archicomble, ils ne purent trouver que des places debout, près d'une colonne, à trois mètres de l'orateur. Tous les deux sont frappés par l'apparence physique de Rathenau, son allure aristocratique, le raffinement de son élégance, l'intelligence de ses yeux ${ }^{15}$. Sa venue à la tribune suffit à installer le silence dans la salle. En outre - et c'est une surprise pour Salomon qui avait donc des préjugés - l'éloquence de l'orateur est sincère, empreinte, certes, de pathos, mais le ton sonne juste ${ }^{16}$, l'émotion est vraie, la profondeur du deuil ne trompe pas. Rappelons que ce discours de Rathenau visait à protester contre le partage de la Silésie que venait de décider la SDN et qui était particulièrement défavorable pour l'Allemagne. Certes, Salomon regrette que la protestation de Rathenau ne jaillisse pas essentiellement de la blessure du sentiment national, mais seulement de la violation d'un principe, le principe de justice. Mais cette ombre a sa lumière, cette réserve a pour contrepartie une qualité précieuse dans la mesure où elle révèle chez cet homme l'existence d'un idéal moral, d'une éthique ${ }^{17}$. 
Pour Salomon et ses amis c'est là une exception de taille dans le personnel politique de l'époque.

11 Ce discours de Francfort n'est pas le seul discours de Rathenau qu'ils ont eu l'occasion d'entendre. Alors que les préparatifs de l'assassinat sont déjà bien avancés, Kern et Fischer, les deux meneurs du groupe, se rendent au Reichstag pour écouter un des grands discours de Rathenau. S'agirait-il de son discours du 23 juin 1922 ? Salomon ne précise davantage. On peut d'ailleurs se demander s'ils n'allaient pas plutôt écouter les interventions des députés du Parti national-allemand et plus particulièrement le réquisitoire très violent que l'un de ses leaders, Helfferich, lança justement la veille de l'attentat contre la politique étrangère de Rathenau. Quoi qu'il en soit, revenant du Reichstag, nos deux amis s'arrêtent devant la devanture d'un photographe où est exposé un portrait de Rathenau. Ils se sentent quasiment interpellés par les yeux sombres, chaleureux, recueillis du ministre et, après une longue hésitation, Fischer finit par déclarer : « Il a l'air très bien $»^{18}$.

Dans le chapitre consacré à la Haute-Silésie est évoqué un discours antérieur de Rathenau, celui du 22 mai 1921 où il protestait contre l'invasion polonaise en Silésie, le viol de la Silésie, écrit Salomon. Le discours, assez bref, est cité intégralement et le mot « viol » revient à deux reprises. Ce détail indique clairement que Salomon se réclame de Rathenau comme d'une caution éminente. D'autres éléments le donnent à penser. Rathenau qui avait placé sa protestation sous le signe du respect des traités et de la justice concluait son intervention par un avertissement exprimé dans les vers fameux de Guillaume Tell par lesquels Schiller invite le peuple à la révolte et à la reconquête par la force de ses droits inaliénables dès lors qu'ils sont foulés aux pieds ${ }^{19}$. Salomon reprend ensuite immédiatement le fil de son récit pour rapporter que le jour même où fut élevée cette noble protestation de jeunes nationalistes combattaient ardemment en Silésie, cependant que le corps franc Oberland reprenait aux Polonais la ville d'Annaberg. Alors que les jeunes insurgés attendaient un ordre du gouvernement pour parfaire une victoire alors à portée de la main, ce dernier, intimidé par une note de Briand qui exigeait la dissolution des formations d'autodéfense, publia un décret qui condamnait et punissait toute action militaire entreprise sans son accord. Bref, un aveu d'impuissance. On comprend alors très bien pourquoi ce discours de Rathenau est cité intégralement : traduisant un sursaut de la conscience patriotique, il offrait aux jeunes nationalistes une justification de leur entreprise. Avant de le citer, le narrateur avait précisé qu'il avait été tenu huit jours avant l'entrée de Rathenau dans le premier cabinet du chancelier Wirth comme ministre de la Reconstruction. Ainsi suggère-t-on au lecteur qu'avant sa prise de fonctions Rathenau pouvait passer aux yeux des jeunes nationalistes pour une personnalité tout à fait respectable et que le projet de son assassinat n'aurait pris corps qu'à partir du moment où, reniant ses engagements antérieurs, il avait accepté de collaborer au sein d'un gouvernement couard et indigne.

Salomon s'applique aussi à souligner qu'avant de découvrir l'homme Rathenau et ses premières interventions politiques, les «Réprouvés » avaient une connaissance précise de ses écrits. Il ne fait guère de doute qu'il veuille ainsi infirmer le reproche d'inculture formulé par plusieurs observateurs lors du procès. Dans sa chronique, il se présente luimême comme le diffuseur des œuvres de Rathenau auprès de ses compagnons et notamment de leur chef de file, Kern.

Pour sa part, Salomon assure avoir rencontré l'auteur Rathenau dès l'âge de dix-sept ans, à un moment particulièrement propice pour lui. Après les troubles de novembre 
1918, il se trouve subitement coupé de cette communauté guerrière où il se sentait si à l'aise. Désemparé, il cherche un refuge et une solution dans les livres avant de repartir à l'aventure comme Parsifal ou Simplicius Simplicissimus auxquels il se compare en toute modestie. C'est alors qu'il remarque dans une devanture de librairie, un peu empoussiéré, le livre le plus célèbre de Rathenau, Von kommenden Dingen, paru en 1917. Ce titre alléchant agit sur lui comme un appel. Il l'achète tout aussitôt et consacre toute la nuit suivante à le lire dans des conditions d'inconfort particulièrement difficiles, ouvert à la réception d'une influence.

Le titre, néanmoins, ne va pas tenir ses promesses. Salomon va sortir de sa lecture avec des sentiments ambigus. Si deux éléments l'ont particulièrement séduit, l'idéalisme du contenu et la simplicité du style, l'ouvrage lui semble à la fois " profond et sans poids » ${ }^{20}$, incapable en tout cas de dispenser la moindre leçon d'enthousiasme ou le moindre message mobilisateur. Malgré la virtuosité de l'analyse, le lecteur a le sentiment de traverser un rêve embrouillé qui n'aboutit pas. Les perspectives proposées ont nom liberté et démocratie, c'est-à-dire, aux yeux de Salomon, des poncifs vociférés par les nantis. Ce livre enfin qui n'est pas à la hauteur des questions qu'il pose et des nostalgies qu'il éveille dans son ouverture est " réactionnaire par l'esprit $»^{21}$ dans la mesure où la critique du présent qu'il contient n'est destinée qu'à conforter l'état de choses existant. Critique conservatrice et non radicalement destructrice ou novatrice. Ce n'est pas un prophète qui s'exprime ici, mais un aveugle qui ne peut donc pas proposer de solutions aux questions posées par l'urgence de la situation historique.

Après cette brève halte, Simplicius reprend sa route. Qu'on n'aille surtout pas en conclure qu'il s'apprête à renier les livres. Bien au contraire. L'action clandestine se double d'une aventure intellectuelle. Salomon est alors saisi d'une véritable frénésie de lecture. Il suit les cours d'un centre de culture populaire. Il fréquente l'université pour compléter ses connaissances en économie. Il s'intéresse à l'histoire des religions. Il s'adonne aux cultes solaires dans le Taunus. Il dévore Nietzsche. Dans sa mansarde, sur des étagères de fortune qu'il a bâties lui-même avec des planches de caisses de grenades, Von kommenden Dingen voisine pêle-mêle avec les œuvres de Marx, de Nietzsche, de Stendhal, de Dostoïewski, de Langbehn. Vitrine éloquente de cosmopolitisme culturel. Von kommenden Dingen n'échappe pas à l'attention de Kern qui, loin de condamner ce choix chez son ami, demande à emprunter le livre et ne tarde pas à le lui rendre accompagné d'un verdict qui rejoint celui de Salomon, à ceci près qu'il est exprimé dans le langage lapidaire et percutant de l'activiste: "Tant d'étincelles pour si peu de dynamite $! »^{22}$.

À travers ce commentaire d'une œuvre de Rathenau, Salomon tient donc à récuser le reproche d'inculture adressé par les témoins de l'époque. Par la même occasion, il nous démontre aussi que ses amis et lui-même, tout en étant sensibles à la qualité et au brio de l'écrivain, ne pouvaient trouver en lui un inspirateur. Reste à expliquer pourquoi la figure de cet inspirateur manqué allait leur devenir insupportable au point de justifier son assassinat. Les raisons sont de nature politique et relèvent bien d'une idéologie de la haine, même si, là aussi, Salomon apporte beaucoup de soin à le dissimuler.

Dans Les Réprouvés, Rathenau n'apparaît pas seulement comme homme, comme orateur, comme écrivain, mais aussi comme l'avocat le plus brillant d'une politique que les "Réprouvés " abhorraient: la politique dite d'accomplissement, c'est-à-dire d'exécution des conditions fixées à l'Allemagne par le traité de Versailles. Salomon fait précéder l'assassinat de Rathenau de toute une série de débats dans lesquels il se 
présente lui-même comme le contradicteur de Kern et où le pour et le contre est soigneusement pesé. Comprenons que l'attentat n'a pas été le fruit d'une réaction d'hostilité viscérale ou la mise en œuvre machinale d'un ordre lancé du dehors par une organisation clandestine. Il a été précédé d'un procès en bonne et due forme à l'issue duquel Rathenau a été condamné.

La première raison de la condamnation tient aux fondements économiques de son attitude politique. À leurs yeux, Rathenau accorde le primat aux facteurs économiques dans la détermination et la conduite de son action ministérielle. Ce faisant, pour Kern, il se soumet à une "tyrannie ${ }^{23}$ dont les conjurés récusent la légitimité. La suprématie reconnue à ces forces matérielles constitue pour Kern la ligne de partage absolue entre eux et le type d'homme politique incarné par Rathenau. Car elle amène fatalement à parler le langage de l'adversaire, à composer avec lui et à rechercher la conciliation, un objectif, on le sait, qui était bien celui du ministre des Affaires étrangères. "Ceux qui parlent d'entente parlent aussi de réconciliation ${ }^{24}$, tranche brutalement Kern en réponse à une interrogation de Salomon. Bien que dans le livre ces réflexions ne soient pas reHées directement à la personne de Rathenau, l'allusion est transparente. Le même passage évoque les diplomates qui se rendent en avril 1922 à la Conférence de Gênes, une conférence consacrée aux questions de l'économie internationale. Or, si la délégation allemande était conduite par le chancelier Wirth, Rathenau, par ses discours et ses entretiens, en fut rapidement le représentant le plus en vue, surtout après la conclusion surprise du traité de Rapallo entre l'Allemagne et l'Union soviétique qui allait tant émouvoir les chancelleries européennes. Par ailleurs, lorsque Kern souligne que ces diplomates adoptent les arguments de l'adversaire et croient trouver dans l'intégration de l'Allemagne au sein du système européen et occidental le remède aux préjudices entraînés par le traité de Versailles, comment ne pas reconnaître là des thèmes que Rathenau avait inlassablement développés tout au long de ses discours? L'intégration à l'Occident, voilà bien le crime suprême. La soumission aux réalités économiques vise à réinstaller l'Allemagne au même rang que les autres puissances, ce qui est une illusion puisqu'elle vient d'être vaincue. Une telle politique dictée essentiellement par des considérations économiques revient à la priver de son identité. Elle équivaut à une perte de substance nationale ${ }^{25}$. Elle constitue bien une trahison.

L'idée de nation se trouve également trahie par le deuxième fondement sur lequel repose l'attitude politique de Rathenau, à savoir le fondement moral dans la mesure où, s'il est respectable, il reste parfaitement gratuit et stérile. Une critique que Salomon avait déjà formulée dans le commentaire du discours prononcé à Francfort, lorsqu'il notait que face aux événements de Silésie l'orateur traduisait davantage la blessure de l'homme moral que celle du patriote. Rathenau est alors présenté comme l'homme politique qui incarne le mieux la conjonction de l'humanisme et du capitalisme, autrement dit la démocratie bourgeoise traditionnelle. Selon Salomon, ce n'est pas un hasard si le charme de son discours opère quasi magiquement sur un public francfortois. Car l'esprit de la ville de Francfort est marqué par une double polarité symboliquement représentée par les deux hommes qui ont particulièrement contribué à son renom : Goethe et Rothschild ${ }^{26}$. Cette union qui est pour Salomon l'emblème par excellence du libéralisme fournit la clef de l'identité politique de Rathenau. Recourant à nouveau au procédé qui veut que la victime fournisse à ses assassins la meilleure des cautions, Salomon évoque une de ses réflexions extraite de Zur Kritik der Zeit ${ }^{27}$ selon laquelle l'homme d'État devait réaliser en lui la synthèse de la force et de l'habileté diplomatique, du mal et du bien, être «comme Napoléon et Bismarck, moitié romain, 
moitié levantin, moitié Baldur, moitié Loki $»^{28}$. Séduisante, la conjonction de l'humanisme et du capitalisme est inopérante et stérile. Nous avons vu que Salomon faisait suivre le discours de Rathenau sur la Silésie de l'évocation des sacrifices consentis sur le terrain par les jeunes nationalistes des corps francs. Ces jeunes patriotes révoltés ne se sont pas contentés de composer artistement un beau discours moral assorti de nobles révérences aux valeurs éternelles. Ils ont agi. Grâce à leur action, les deux tiers de la province ont été sauvés. Là se situe la faille décisive d'une stratégie politique telle que l'incarne Rathenau. Une telle attitude ne peut prolonger ses discours en actes, car, troisième moment de la démonstration, elle repose sur un fondement psychologique vicié dans la mesure où elle est liée à une psychologie de la peur.

Pour la définir, Salomon, toujours soucieux de nous montrer qu'il connaît bien son Rathenau, s'abrite une fois de plus derrière l'une de ses œuvres, Reflexionen, pour reprendre la distinction d'ailleurs fort ambiguë qu'il avait établie entre «les êtres de courage et les êtres de peur» (Furcht- und Mutmenschen) ${ }^{29}$. Or, selon Salomon, si on applique cette typologie à Rathenau, les choses sont claires : il faut le ranger parmi « les êtres de peur " comme le trahissent ses intonations, ses gestes, les palpitations de son $\operatorname{regard}^{30}$. Ainsi s'explique que pour lui l'heure est à la négociation et non à l'action héroïque. La distinction établie par la typologie de Rathenau se prêtait d'autant mieux à la démonstration qu'il identifiait les êtres de courage aux Germains. Avant d'avoir été assassiné, Rathenau a été démasqué.

Ayant radiographié Rathenau à partir de ses propres déclarations ou de ses propres écrits, Salomon n'a aucun mal à démontrer que la politique d'accomplissement débouche fatalement sur le renoncement dans la mesure où elle n'est pas animée par la volonté de traduire en actes les principes dont elle se réclame.

Je ne peux croire, commente Kern, qu'une génération comme la nôtre, projetée dans un combat qui l'a éduquée et endurcie, puisse être désormais vouée à renoncer servilement à son combat, comme l'y invite le dérisoire appel de ceux qui reculent, effrayés, devant les conséquences de leurs propres volontés ${ }^{31}$.

Allusion parfaitement claire, car immédiatement avant Kern avait brocardé en la retournant la célèbre phrase que Rathenau avait formulée dans son opuscule intitulé Der Kaiser ${ }^{32}$ sur l'histoire " qui aurait perdu son sens, si l'empereur Guillaume II avait franchi en vainqueur la Porte de Brandebourg sur son blanc destrier, escorté de tous ses paladins ». Pour Kern et ses amis, la défaite n'a pas dévalué le nationalisme qui reste la finalité de l'Histoire. C'est au contraire la politique d'accomplissement qui la prive totalement de sens. Aussi lui oppose-t-il une autre forme d'accomplissement, l'accomplissement par l'action au service de la nation en indiquant clairement que l'objectif d'un tel activisme n'est autre que « le triomphe de la germanité sur la terre » 33. L'opposition radicale des conjurés est donc bien politique et non raciste. À une politique bourgeoise, idéaliste et passive d'êtres apeurés, prudents et calculateurs s'oppose une politique nationaliste et résolue d'êtres jeunes et intrépides. Conflits de générations certes, mais aussi d'idéologies.

Toutefois Salomon n'en reste pas à cette démonstration de type syllogistique qui présenterait un caractère trop simpliste. En outre, il faut bien expliquer pourquoi les conjurés ont choisi de s'en prendre à Rathenau plutôt qu'à un autre représentant en vue des milieux dirigeants. C'est qu'une qualité supplémentaire isole et distingue le ministre des Affaires étrangères du reste du personnel politique. Avocat et théoricien 
de la politique d'accomplissement, il peut aussi en être le maître d'œuvre et la mener à bien. Interrogé par Salomon sur la question de savoir si Rathenau n'a pas mené une politique active, Kern le confirme et reconnaît qu'entre ses mains ont été déposées bien plus de choses que ce ne fut jamais le cas depuis 1918. Il incarne l'espoir ${ }^{34}$. C'est pourquoi il faut lui barrer la route avant qu'il soit trop tard. Dans la présentation de Salomon, Rathenau devient alors une sorte de héros, le héros de la démocratie bourgeoise, humaniste, libérale face au héros de la nation, son ultime recours ${ }^{35}$. Cette qualité scelle son arrêt de mort. Kern confie qu'il ne supporterait pas de le voir triompher. Le héros de la nation et le champion de la démocratie ne peuvent coexister. L'un d'eux doit disparaître. La transfiguration de Rathenau fait planer sur l'attentat, sa genèse et ses développements le climat implacable et pur de la tragédie.

Si la dimension héroïque prêtée à Rathenau dresse irrémédiablement l'un contre l'autre le champion de la démocratie et le héros de la nation, elle crée aussi entre le bourreau et la victime un lien de parenté mystérieuse que révélait, en un raccourci fulgurant, un incident du discours de Francfort, signe avant-coureur de la catastrophe. «Et alors se produisit l'incompréhensible $»^{36}$, écrit Salomon. Au moment où Rathenau abordait le thème de la confiance qui doit entourer le chef, Salomon, dans un éclair de révélation qui ouvrait une porte sur la mort, vit son ami Kern fixer l'orateur d'un regard si intense et si dur que ce dernier en fut profondément troublé, devenant soudain hésitant et cherchant ses mots. Dès lors, il ne put poursuivre son discours que les yeux plongés dans ceux de Kern. Kern devenait son interlocuteur privilégié, puisque Rathenau ne s'adressait plus qu'à lui seul, mais cet interlocuteur restait inaccessible puisque les arguments du ministre n'avaient aucune prise sur lui. Il planait là comme une atmosphère chevaleresque. Loin au-dessus de la foule des auditeurs, Rathenau et Kern sont isolés dans le cercle d'un combat singulier dont le cours aurait pu basculer ce jourlà et qui, bien entendu, n'a eu qu'un seul témoin privilégié : Salomon, On ne peut entraver la marche inexorable du destin. À la fin du meeting, après une ultime hésitation, Kern passe à côté de Rathenau sans s'arrêter et sans relever le regard interrogateur que ce dernier lui lance. Tous les deux, Kern et Rathenau, sont finalement étrangers à la société bourgeoise, Kern en raison de son nationalisme intransigeant, Rathenau en raison des qualités exceptionnelles qu'il met à son service.

Frères ennemis dans la vie, Kern et Rathenau le resteront dans la mort, réunis par la commune incompréhension qui va frapper leur action et leur sacrifice. La société et l'État se montreront tout d'abord incapables de lui organiser des funérailles dignes de sa grandeur. "Rathenau est solitaire également dans la mort $\|^{37}$. Son exemple restera sans postérité et le peuple va continuer de s'enliser dans la médiocrité et la vulgarité à l'exemple de ces trois voyageurs de commerce que Salomon, traqué par la police, côtoie dans le train et qui se racontent des plaisanteries douteuses. L'une d'elles ${ }^{38}$ concerne le ministre assassiné qui, au ciel, rencontre un autre ministre, victime des mêmes milieux : Erzberger. Ce dernier veut fêter leurs retrouvailles avec une bouteille de vin. Mais saint Pierre refuse de les servir, car l'aubergiste (Der Wirth) n'est pas encore arrivé. Incapable de rendre à Rathenau les hommages qu'il mérite, la société est tout aussi incapable de comprendre les mobiles des meurtriers. Et d'abord le premier de ses représentants, Ebert qui, dans son discours prononcé au Reichstag le jour des funérailles, les vouait à la mort ${ }^{39}$. Sans aucun commentaire, Salomon fait suivre le texte d'Ebert d'un extrait de Rathenau tiré de Zur Mechanik des Geistes ${ }^{40}$, dans lequel, à travers des métaphores empruntées à la vie de la nature, l'auteur développait l'idée que dans le 
monde visible la mort n'a jamais le dernier mot et que l'effacement de certains éléments conditionne la perpétuation de l'ensemble. Le but de la citation est clair : contrairement à ce qu'affirme Ebert, la lutte des jeunes nationalistes est appelée à de nouveaux lendemains. Le patronage de Rathenau confère à ce pronostic, qui est peutêtre un vœu implicite, une aura supplémentaire. Seule la victime a compris d'avance ses bourreaux. Seuls les bourreaux ou ceux qui leur survivent peuvent déplorer dignement la victime et tirer des leçons de la tragédie.

Après avoir appris la mort de ses amis Kern et Fischer, Salomon est tenté un moment par le suicide. Une fois de plus, il associe la mort de Rathenau et celle de Kern.

Rathenau était mort et lutter n'avait plus de sens. Kern était mort et vivre n'avait plus de sens ${ }^{41}$.

À la fin du chapitre II, la déploration de Rathenau se clôt sur un examen de conscience qui est une forme de repentir :

Rathenau mourut, et ces gens respectables continuaient de vivre et de s'éclabousser et on ne remarquait pas de vide ${ }^{42}$.

À sa manière, Rathenau, lui aussi, est un réprouvé. La responsabilité de sa mort se dilue au niveau de la société tout entière.

Toute cette présentation est subtile et habile, car la noblesse prêtée à Rathenau rejaillissait partiellement sur ses assassins. Le silence sur l'antisémitisme idéalisait un peu plus leur figure. Le titre de champion ultime de la démocratie bourgeoise conféré à Rathenau comblait les vœux de tous ceux qui entretenaient pieusement son souvenir idéalisé et leur apportait une délectation morose au moment où, en 1930, la démocratie weimarienne était confrontée à une crise démesurée et avait plus que jamais besoin d'un champion.

32 Cette image, cependant, est de mauvaise foi. La part prise par l'antisémitisme dans l'élimination physique de Rathenau est trop délibérément niée pour paraître plausible et les variantes apportées sur ce point par Salomon des Réprouvés au Questionnaire sont plutôt de nature à renforcer les doutes. D'autres doutes se rapportent au soubassement littéraire de cette image. Elle est étayée par des extraits d'œuvres de Rathenau et ces lectures sont données comme antérieures à l'attentat. Est-ce vraiment crédible? Comment admettre sans réticences que Salomon lui-même ait pu découvrir Von kommenden Dingen à dix-sept ans? N'est-il pas invraisemblable qu'il ait pu connaître avant 1922 Zur Mechanik des Geistes, dont la diffusion avait été extrêmement faible ? S'il est tout à fait plausible que Kern ait pu évoquer la fameuse phrase de Der Kaiser sur le sens de l'Histoire à cause de l'intense dénonciation qu'en avait faite les milieux nationalistes, on aura de la peine à croire, en revanche, que les discours de Rathenau aient été aussi familiers aux conjurés, notamment ceux qui avaient été prononcés au Reichstag ? N'y a-t-il pas d'ailleurs une contradiction entre cette mention et le passage ${ }^{43}$ où Salomon nous dit que lui et ses amis étaient depuis longtemps étrangers aux délibérations des parlements et à tous les rites de la politique officielle? Toutes ces lectures ont sans doute été faites après coup, pour Salomon durant les années de prison, comme il l'explique d'ailleurs dans Le Questionnaire:

Je lus tous les ouvrages de l'État-major de la Grande Guerre, les dossiers du ministère des Affaires étrangères, tout Jean-Paul, Dickens, Raabe, Stifter, Dostoïewski, Thackeray et naturellement toutes les œuvres de Walther Rathenau... 44 . 
N'est-il pas curieux enfin que Salomon, lorsqu'il se réclame de la caution littéraire de Rathenau, ne retienne presque toujours de ses réflexions que celles qui sont effectivement les plus contestables?

D'un livre à l'autre on relève aussi des contradictions entre les différentes versions qu'il donne des motivations de l'attentat. Dans Les Réprouvés, il fait nettement ressortir que la mort de Rathenau a été décidée par les conspirateurs eux-mêmes, en toute indépendance, hors de toute allégeance à une organisation extérieure, notamment de «l'organisation Consul ». Dans Le Questionnaire, si l'entrevue orageuse des conjurés avec le capitaine Ehrhardt ${ }^{45}$, qui était à la tête de cette organisation et condamne violemment l'attentat, paraît confirmer cette thèse, il évoque auparavant ${ }^{46}$, tout en minimisant d'ailleurs sa portée, l'existence d'une liste de personnalités à abattre sur laquelle figurait le nom de Rathenau et qui serait tombée mystérieusement entre leurs mains. On est loin ici du climat chevaleresque et de l'hommage rendu à la grandeur de l'adversaire.

35 Il arrive enfin à Salomon de pécher par omission quand il se garde, par exemple, d'évoquer certains éléments gênants pour la cohérence de sa démonstration. Ainsi, dans le chapitre des Réprouvés intitulé "Aktion » ${ }^{47}$, il évoque la fureur du peuple allemand devant cette disposition du traité de Versailles qui exigeait l'extradition des criminels de guerre, c'est-à-dire de certains dirigeants jugés responsables de la prolongation de la guerre. Mais il oublie de mentionner que Rathenau avait figuré initialement sur la première liste qui avait été rendue publique. Le patriotisme ne doit pas figurer parmi les signes distinctifs de Rathenau.

L'image de Rathenau brossée par Salomon dans Les Réprouvés et Le Questionnaire est une reconstruction, un montage savamment agencé. Dans sa préface de 1961 au Questionnaire, Salomon se défendait d'avoir voulu entreprendre une justification de l'attentat. Il s'en garde bien en effet, mais la présentation qu'il en fait est fortement teintée de sympathie à l'égard des assassins, ce qui peut se comprendre, mais aussi à l'égard de leur victime, ce qui éveille quelques soupçons. Quand le bouc émissaire est transfiguré à ce point en héros, quand les racines ethniques de l'attentat sont si délibérément niées, on ne peut s'abuser sur la finalité de cette image légendaire et trompeuse, construite pour les besoins d'une cause que Salomon n'a jamais vraiment reniée, même s'il s'est tenu à l'écart du nazisme : la haine des valeurs démocratiques. «Si d'horribles circonstances contraignent des Européens à s'opposer à je ne sais quels périls, Les Réprouvés seront leur bréviaire ", écrit encore Roger Stéphane à la fin de l'article déjà cité. Il nous paraît difficile de souscrire à un tel jugement, car les leçons que délivre ce livre, au-delà de son indéniable valeur documentaire, nous paraissent bien fallacieuses et fort éloignées d'un code d'honnête conduite. 


\section{NOTES}

1. - En France, Les Réprouvés ont été réédités en 1986 chez Christian Bourgois, coll. "10/18 ", traduction d'Andrée Vailland et Jean Kuckenburg, ainsi que Les Cadets, traduction d'Edmond Lutrand et Eve Dessarre.

2. - CEuvres citées d'après l'édition allemande Rowohlt. Rororo, Die Geächteten, 1962 = abréviation DG ; Der Fragebogen, 1951, Rowohlt Ferlag = abréviation DF.

3. - Roger Stéphane, Le Monde, 31 janvier 1986 : « Retrouver Ernst von Salomon. On réédite Les Cadets et Les Réprouvés : une œuvre essentielle pour comprendre l'Allemagne ».

4. - DG, p. 218.

5. - Ibid., p. 150.

6. - Voir P.-P. Sagave, Études germaniques, 9, 1954, et 10, 1955 : « Ernst von Salomon. Son milieu, ses idées, ses récits ".

7. - Ernst Feder, Heute sprach ich mit... Tagebücher eines Berliner Publizisten (1926-1932), Stuttgart, Deutsche Verlags-Anstalt, 1971.

8. - Ibid., p. 221, Mittwoch, 24 juillet 1929.

9. $-D F$, p. $128-129$.

10. - W. Hartenau, Höre Israël !, in Die Zukunft, 18, 1897, p. 454-462.

11. - W. Rathenau, Briefe I, 5 février 1915, 23 janvier 1916, 25 janvier 1916, 18 août 1916, 16 mars 1919.

12. $-D F$, p. 129.

13. - Ibid., p. 129.

14. - Ibid., p. 129.

15. - DG, p. 190.

16. - Ibid., p. 190.

17. - Ibid., p. 190-191.

18. - Ibid., p. 217.

19. - Ibid., p. 167-168. «Wenn der Gedrückte nirgends Recht kann finden, / Wenn unerträglich wird die Last - greift er / Hinauf getrosten Mutes in den Himmel / Und holt herunter seine ew'gen Rechte, / Die droben hangen unveräusserlich / Und unzerbrechlich wie die Sterne selbst.»

20. - Ibid., p. 142.

21. - Ibid., p. 143.

22. - Ibid., p. 157.

23. - Ibid., p. 210.

24. - Ibid., p. 210.

25. - Ibid., p. 210.

26. - Ibid., p. 191.

27. - W. Rathenau, Zur Kritik der Zeit, Berlin, Fischer, 1912, p. 225.

28. - DG, p. 191.

29. - Ibid., p. 191.

30. - Ibid., p. 191.

31. - Ibid., p. 208.

32. - W. Rathenau, Der Kaiser, Berlin, Fischer, 1919, p. 305.

33. - DG, p. 209.

34. - Ibid., p. 212.

35. - Ibid., p. 213.

36. - Ibid., p. 192. 


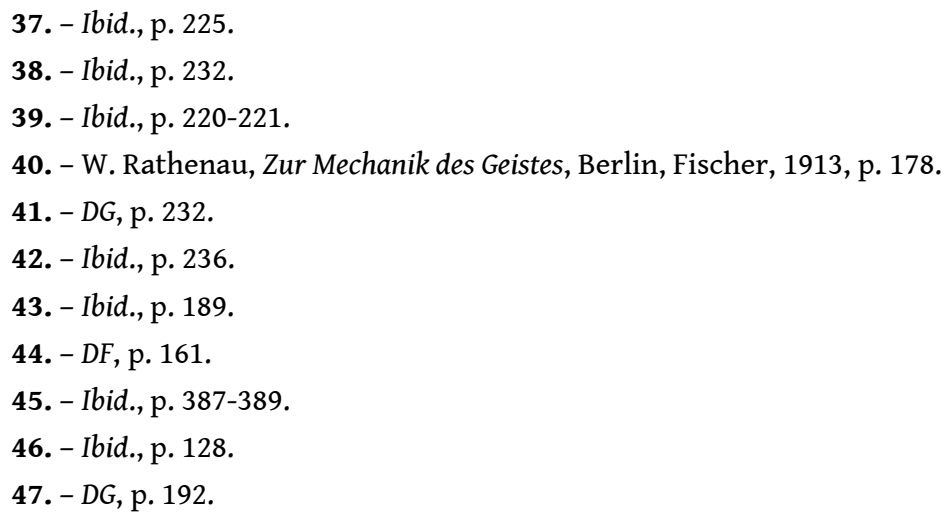

\section{RÉSUMÉS}

Dans deux de ses œuvres autobiographiques, Les Réprouvés et Le Questionnaire, Ernst von Salomon, qui s'est trouvé mêlé à l'assassinat de Rathenau, bouc émissaire des milieux nationalistes, nie délibérément les racines ethniques de cet acte. L'attentat n'aurait été ni le fruit de l'antisémitisme ou de la haine instinctive, ni l'exécution machinale par des êtres frustes d'un ordre venu d'ailleurs. La victime fait l'objet d'une transfiguration qui ne manque pas d'intriguer. Cette présentation fallacieuse, souvent contradictoire et toujours sélective, est le résultat d'un montage savamment agencé.

In zwei seiner autobiographischen Werke, Die Geächteten und Der Fragebogen, leugnet Ernst von Salomon, der in die Ermordung Walther Rathenaus, eines Sündenbocks der nationalistischen Kreise, verwickelt wurde, ausdrücklich die ethnischen Beweggründe dieser Tat. Das Attentat sei weder die Frucht des Antisemitismus bzw. eines instinktiven Hass gefühls noch die maschinelle Ausführung eines von höherer Warte diktierten Befehls durch grobschlächtige Handlanger gewesen. Das Opfer wird zum Gegenstand einer Verklärung, die notwendigerweise befremdlich wirkt. Diese trügerische, häufig widersprüchliche une immer selektive Darstellung ist das Endergebnis einer gekonnt durchgeführten Montagetechnik.

In two of his autobiographical works, The Outlaws and The Answers, Ernst von Salomon, who was involved in the murder of Rathenau (the scapegoat of the nationalists), vigorously denies the racial basis of this action. The murder is said not to have been motivated by antisemitism, instinctive hatred, or the mechanical execution of superior orders. Thus the victim undergoes a quite puzzling metamorphosis. This spurious demonstration, which is often self-contradictory and constantly biased, is the result of a careful and intelligent manipulation.

\section{AUTEUR}

\section{PIERRE GIRAUD}

Université de Lille III 